\title{
Magnetic stray fields of Co-Cr microstrips measured by Lorentz microscopy
}

\author{
J. G. Th. te Lintelo and J. C. Lodder \\ Mesa Research Institute, University of Twente, P.O. Box 217, 7500 AE Enschede, The Netherlands
}

S. McVitie and J. N. Chapman

Department of Physics and Astronomy, University of Glasgow, Glasgow G12 $8 Q Q$ United Kingdom

(Received 6 May 1993; accepted for publication 6 December 1993)

\begin{abstract}
Magnetic stray fields of $\mathrm{Co}-\mathrm{Cr}$ microstrips are investigated for bit stabilization in a vertical Bloch line memory (VBLM). The stray fields were revealed using Lorentz force based Foucault and differential phase contrast (DPC) techniques in a transmission electron microscope. The assumed shape of the stray fields for VBLM use has been experimentally verified. Agreement between experiment and simulation is obtained.
\end{abstract}

\section{INTRODUCTION}

In the study of the vertical Bloch line memory $(\mathrm{VBLM})^{1}$ much progress has recently been made by experimentally demonstrating the feasibility and compatibility of different device functions on a 1 kbit prototype chip. ${ }^{2}$ However the stability and propagation of bits (i.e., VBL pairs) in the minor loop is still a point of concern.

VBLs can be stabilized by an in-plane magnetic stray field along stripe domains according to

$$
H_{i p}=H_{i p 0} \cos \left(\frac{x}{2 \Lambda_{0}}\right),
$$

where $\Lambda_{0}$ is the Bloch line width parameter and $H_{i p 0}$ is in the order of $0.5 \mathrm{kA} / \mathrm{m} .{ }^{3}$ The $x$ direction is pointing along the domain wall of the garnet. Technologically, this can be achieved by different methods, ${ }^{4}$ however, an overlay of hard magnetic strips is thought to be the most practical solution for power consumption and bit density concerns. ${ }^{5}$

In this article Lorentz microscopy observations that investigate the actual stray fields emerging from $\mathrm{Co}-\mathrm{Cr}$ microstrips are reported. Knowledge of the (bit stabilizing) magnetic field is important because its form and amplitude define the physical bit locations. The strips used in the present study are the same as those investigated in a previous study ${ }^{6}$ that concentrated on the change in magnetic properties as a function of the shape of $\mathrm{Co}-\mathrm{Cr}$ samples and their relationship to the demagnetizing factor. The aim of the present study was to observe whether the magnetic stray fields behave according to the form of Eq. (1).

The experimental conditions for these observations are described in Sec. II, whereas the observations themsclves may be found in Sec. III. In Sec. IV a correlation to theory is made.

\section{EXPERIMENT}

\section{A. Preparation}

Co-Cr films were if sputtered from alloyed targets $(\mathrm{Cr}$ content: 19,21 , and 23 at. \%) under optimized conditions. ${ }^{7}$ Photolithography was performed using a positive resist (Shipley S1400-31), and ion beam milling $\left(V_{a c c}=500 \mathrm{~V}, I_{\text {cath }}=12.5 \mathrm{~mA}\right.$, Ar flow $\left.=200 \mathrm{sccm}\right)$, which caused no annealing effects on the Co-Cr. The strip width was $\sim 5 \mu \mathrm{m}$ and the period varied $(8,10,15$ and $20 \mu \mathrm{m})$. All samples exhibited a uniaxial anisotropy perpendicular to the substrate that was larger than the (diminished) demagnetization $(Q>1)$. A more detailed description of the magnetic properties is given in Ref. 6. In Fig. 1 the geometry of the strips and the definition of the parameter notation are given. In Table I some properties are given for the three samples used for this research.

The small samples required for the observations by transmission electron microscopy (TEM) were cut from $1 \times 1 \mathrm{~cm}$ samples. A scanning electron microscope (SEM) photograph is given in Fig. 2 with an example of a strip and its fracture. This particular sample has been etched deeply into the Si substrate.

These samples (typical dimensions: $0.4 \times 0.3 \times 2 \mathrm{~mm}$ ) were glued with silver paint on 3-mm-diam copper grids. This is depicted in Fig. 3, showing that the length of the strips is parallel to the path of the electron beam in the TEM. Usually, the sample had to be tilted to get a good alignment between the electron beam and the strips. A small additional tilt was made around the $x$ axis (see Fig. 3 ) in order to circumvent the influence of small misalignments and some dust inbetween the strips that was caused by the preparation process. This adjustment made it possible to scan between the strips.

\section{B. Lorentz microscopy}

Two modes of Lorentz microscopy were used to reveal the magnetic stray field of the $\mathrm{Co}-\mathrm{Cr}$ microstrips, the Foucault and the modified differential phase contrast method (DPC).$^{8}$ The Foucault mode was implemented in a JEOL $2000 \mathrm{FX}$, with the sample in an essentially magnetic field free space. ${ }^{9}$ In this mode the images of the stray field components perpendicular to the direction of electron beam travel can be obtained in a relatively easy and fast way. In general, this technique was suitable for selecting the most interesting sample for further study.

These samples were investigated in more detail with the DPC mode in an extended VG HB 5 scanning transmission electron microscope (STEM) equipped with a field emission gun. ${ }^{9}$ In this mode the objective lens was 


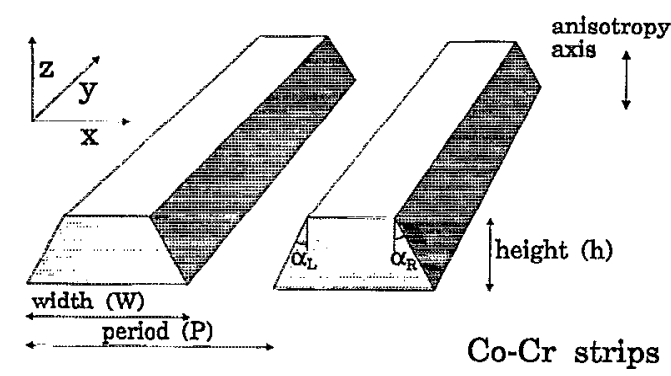

FIG. 1. Geometry of the strips and definition of the parameters notation.

turned off in order to avoid the magnetic fields in the plane of the sample. The image resolution was around $20 \mathrm{~nm}$.

\section{Data processing}

The different intensity levels of Lorentz images can be associated with the varying values of a particular component of the induction in the cross-sectional plane. With a pair of images sensitive to orthogonal components of induction (i.e., the $x$ and $z$ component in Fig. 3), a map of the distribution of the magnetic induction was obtained by a magnetic induction mapping program, described elsewhere. ${ }^{10}$ In addition, line profiles of the different components of the induction were obtained on a Link eXL system and offer the possibility of further quantitative analysis and comparison to modeling.

\section{EXPERIMENTAL RESULTS}

\section{A. Foucault imaging on the JEOL 2000 FX}

Figure 4 shows a typical example of an image with the induction sensitivity parallel to the substrate. The magnetic stray fields can be clearly observed and are consistent with a direction of the magnetization perpendicular to the substrate.

In Fig. 5 a sample identical to that in Fig. 4 reveals more detail near the surface. This image shows regular dark and white regions near the surface of the strips. Such contrast is not observed inbetween the strips or in the normal TEM image, suggesting that it is of magnetic origin. A mean period of $250 \mathrm{~nm}$ is found and is in the theoretical range of a domain period for this type of sample. ${ }^{11}$ Confirmation that this is, indeed, a domain structure is difficult because the image represents the average magnetic stray field intensity of several domains along the length of the strip.

TABLE I. Geometrical, compositional, and magnetic information on the investigated samples.

\begin{tabular}{ccccccc}
\hline \hline $\begin{array}{c}\text { Sample } \\
\text { No. }\end{array}$ & $\begin{array}{c}\text { Sample } \\
\text { name }\end{array}$ & $\begin{array}{c}\text { Thickness } \\
(\mathrm{nm})\end{array}$ & $\begin{array}{c}\mathrm{Cr} \text { content } \\
(\text { at. \%) }\end{array}$ & $\begin{array}{c}M s \\
(\mathrm{kA} / \mathrm{m})\end{array}$ & $\begin{array}{c}H c \\
(\mathrm{kA} / \mathrm{m})\end{array}$ & $\begin{array}{c}K 1 \\
\left(\mathrm{~kJ} / \mathrm{m}^{3}\right)\end{array}$ \\
\hline 1 & 080191 & 825 & 18.6 & 474 & 76 & 118 \\
2 & 250291 & 910 & 19.2 & 447 & 64 & 447 \\
3 & 050391 & 650 & 23.0 & 300 & 54 & 70 \\
\hline
\end{tabular}

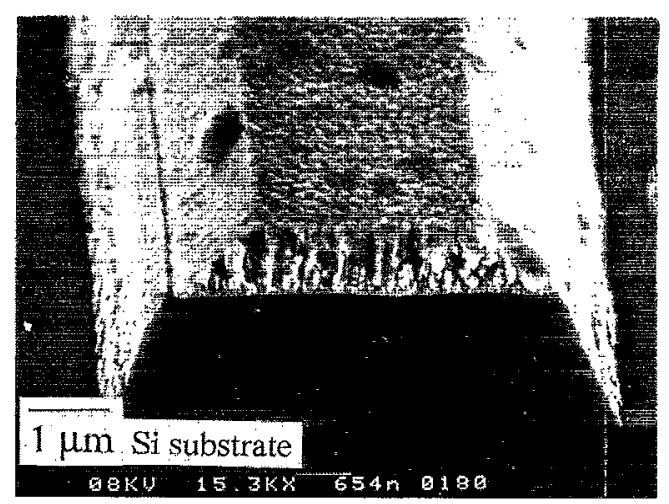

FIG. 2. SEM photograph of a patterned $\mathrm{Co}-\mathrm{Cr}$ strip on the $\mathrm{Si}$ (sample No. 2).

A fine magnetic contrast on top of the strips could not be observed with all of the investigated samples. However, the image in Fig. 4 with its black and white saturated regions is obtained easily. From this, global magnetic information can be obtained. The most interesting samples were determined by these studies and are investigated in more detail in the DPC mode.

\section{B. DPC imaging on the HB 5}

Figure 6 shows a typical example of two images sensitive to orthogonal induction components and obtained by the DPC technique. The magnetic stray fields can again be clearly observed and a fine magnetic contrast is also apparent.

In Fig. 7 an image identical to sample in Fig. 6 is taken, but after applying a strong magnetic field. This is done by switching the objective lens ON and OFF. A difference is evident. Not only the magnetization direction has changed, but also the black and white regions at the surface have changed, proving that the contrast observed in Fig. 6 is, indeed, magnetic. The scale of the domains is consistently less than $1 \mu \mathrm{m}$, and the precise pattern on any particular strip depends on the magnetic field to which it was subjected.

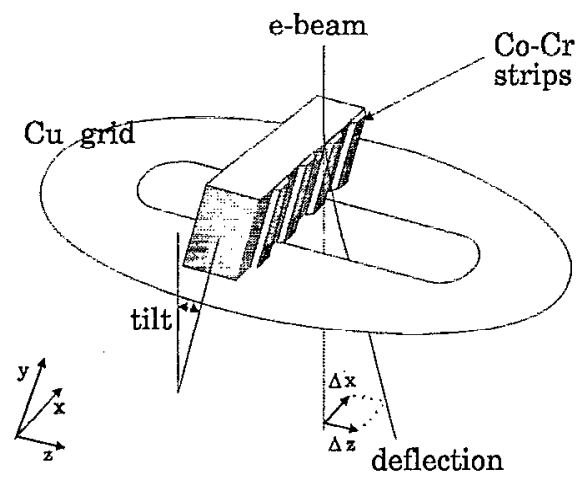

FIG. 3. Deflection of the e-beam by the Lorentz force due to the magnetic stray fields of Co-Cr microstrips. 


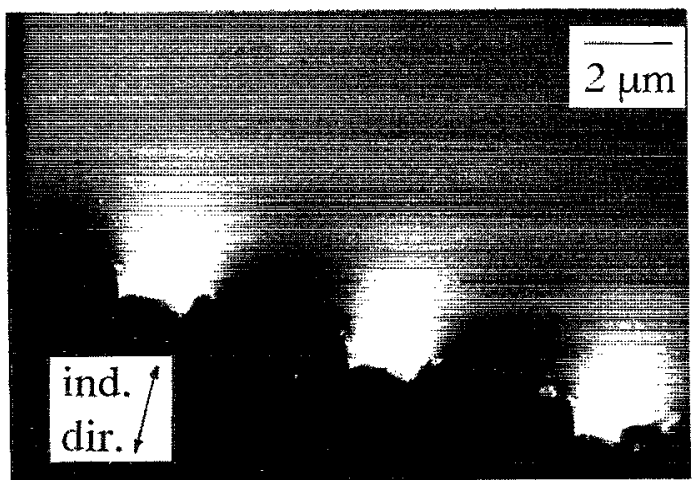

FIG. 4. Foucault image of sample No. 3 with the induction mapped perpendicular to the substrate.

In order to quantify the variation of the component of magnetic induction, line scans were taken. Figure 8 shows three typical examples of line scans taken at different distances from the sample. The line scans in this figure show the required modulation, as described in Eq. (1) for distances larger than $1 \mu \mathrm{m}$. For VBLM a spacer layer of $1 \mu \mathrm{m}$ is proposed ${ }^{12}$ and the results here indicate that this is sufficient for application purposes. However, the similarity in modulation only concerns the shape. No statement can be made on the absolute amplitude because the absolute deflection of the e-beam was not measured. In Fig. 9 the corresponding $z$ component of the magnetic induction is shown.

In Fig. 10 line scans of another sample are shown. The stray field intensity near the surface is different from that in Fig. 9. The line scans further away from the strips, however, again resemble Eq. (1). In Sec. IV this will be discussed further.

The two combined images give insight as to the direction in which the magnetic stray field is pointing. This information can easily be obtained by using the induction mapping program described in Ref. 10. In Fig. 11 an example of the equivalent to that of Fig. 10 is shown. Note that the length of the arrows, which are representing the magnitude of the stray field, are on a logarithmic scale.

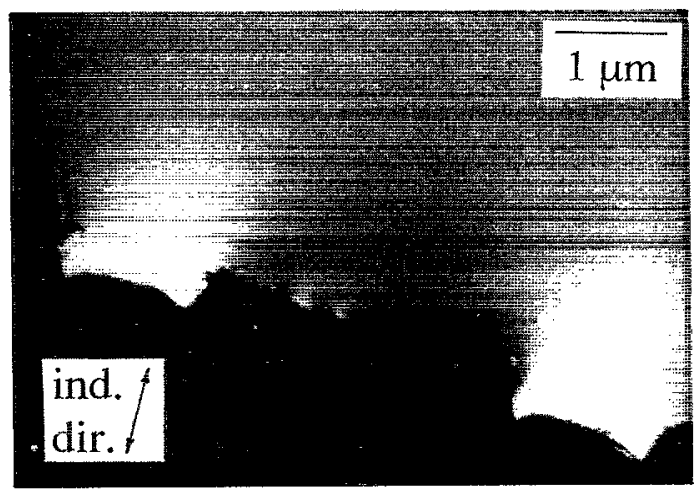

FIG. 5. Foucault image of the sample No. 3 with the induction mapped perpendicular to the substrate.

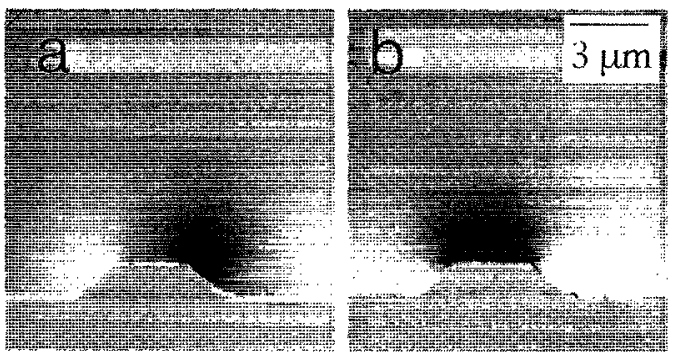

FIG. 6. DPC images of the sample No. 2. Induction mapped parallel to the substrate (a), and induction mapped perpendicular to the substrate (b).

This is done to obtain more information on the field directions. The symmetry can be clearly observed and the resemblance to uniformly magnetized permanent magnets is remarkable.

\section{SIMULATIONS}

Whereas the resemblance of the magnetic stray fields to that expected from uniform magnetized permanent magnets is clear, a simple model was used to correlate the observed effects to theory. This model is based on calculating the magnetic field from the four sides of the strips (including the tilting effect of the side flanks) by considering them as sheets filled with a uniform surface magnetic charge. ${ }^{6}$

In Fig. 12 simulated equivalents of Figs. 8 and 9 are shown. A large similarity can be observed, especially for the largest distances, where the required shape of Eq. (1) is obtained.

The similarity decreases when a vector map is simulated. In Fig. 13 a simulated equivalent of Fig. 11 is depicted. Although both figures show arrows that follow the line of a closing flux path, the theoretical stray field is more or less symmetric at about the line $P-P^{\prime}$ that runs through the center of the strip, as was expected. However, in the experimental situation the symmetry line is shifted outside the strip, towards the silicon substrate. The difference is due to the path of the e-beam. The electron will experience different stray fields along its path due to the tilt and, thus, different distances to the strip. Therefore the magnetic stray fields have to be integrated along the electron

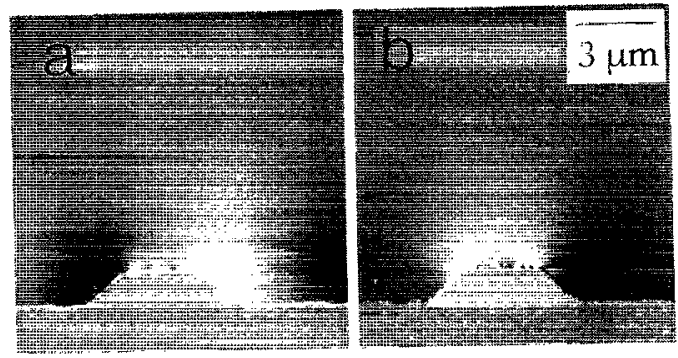

FIG. 7. DPC images of the sample No. 2, after applying a strong magnetic field. Induction mapped parallel to the substrate (a), and induction mapped perpendicular to the substrate (b). 

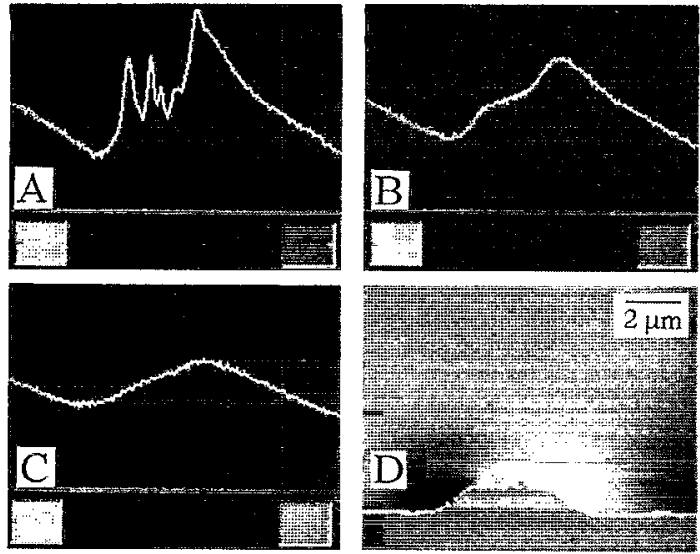

FIG. 8. Intensity line scans of the $x$ component of the magnetic induction for the sample No. 2. Distance to the surface of the strip. $A=0.1, B=0.4$, $\mathrm{C}=1.0 \mu \mathrm{m} . \mathrm{D}=$ bright field image of the same area.

trajectory. ${ }^{8}$ We incorporated them by tilting the sample (see Fig. 14), in the model and by calculating the stray field every $0.5 \mu \mathrm{m}$ along a path that extends to $300 \mu \mathrm{m}$ along the strips and starts $5 \mu \mathrm{m}$ before the beginning of the strip. The tilt in the $z$ direction is the one that is imposed by the experiment (see Sec. II, Fig. 2) and will be of the order of $0.1^{\circ}-2^{\circ}$. A tilt in the $x$ direction, due to small errors in misaligning the strips parallel to the e-beam, will be neglected. The distance to the strip is taken as the distance in the $z$ direction between the e-beam and the uppcr surface of the strip, at the first tip of the strip. This is denoted $\delta$ in Fig. 14.

The Lorentz force is also taken into account. In a normal DPC measurement this angle is smaller than 0.5 mrad, ${ }^{8}$ but this is not true for these experiments. The deflection angle $\beta_{l}$ for electrons experiencing a constant magnetic induction is given by ${ }^{8}$

$$
\beta_{l}=\frac{e \lambda}{h} \int_{-\infty}^{+\infty} B d y=\frac{e \mu_{0} \lambda}{h} \int_{-\infty}^{+\infty} H d y
$$

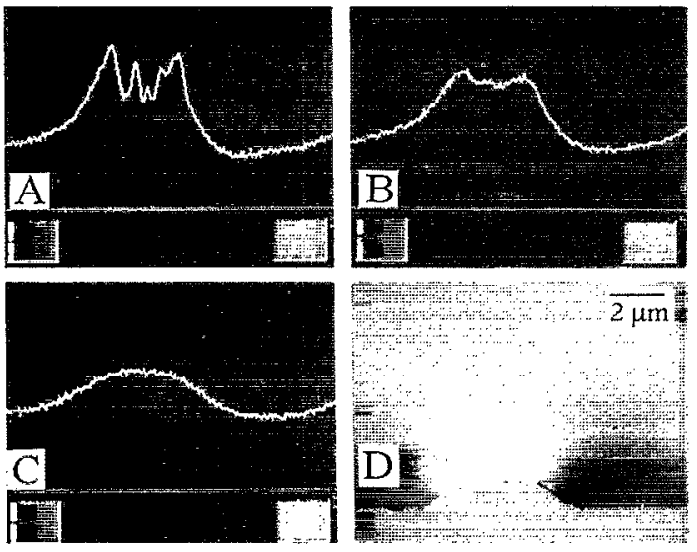

FIG. 9. Intensity line scans of the $z$ component of the magnetic induction for the sample No. 2. Distance to the surface of the strip. $A=0.1, B=0.4$, $\mathrm{C}=1.0 \mu \mathrm{m}$. D = bright field image of the same area.
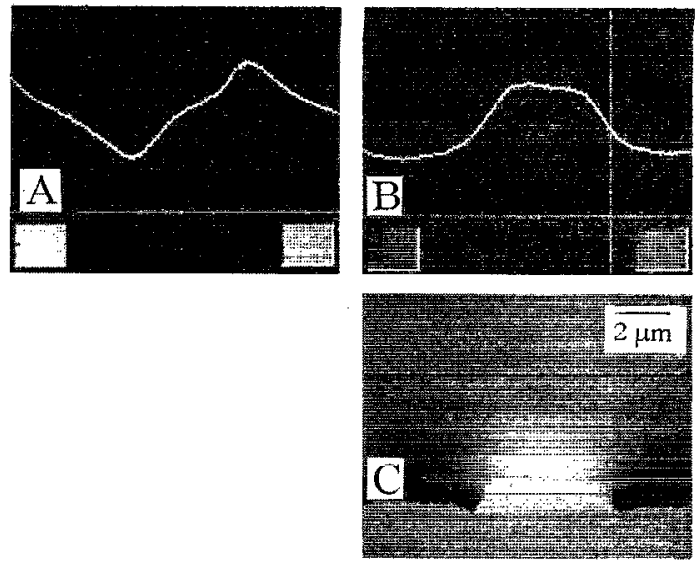

FIG. 10. Intensity line scans of the sample No. 2 with a distance to the surface of the strip of $0.2 \mu \mathrm{m}$. (A) $x$ component of magnetic induction, (B) $z$ component of magnetic induction, (C) bright field image of the same area.

where $e$ is the electron charge, $h$ is Planck's constant, $\mu_{0}$ is the permeability of free space, and $\lambda$ is the electron wavelength. The wavelength in these experiments was $3.7 \mathrm{pm}$. Taking a path of $300 \mu \mathrm{m}$ with an average stray field of 5 $\mathrm{kA} / \mathrm{m}$ (see also Fig. 15) results in a deflection angle of 1.7 $\operatorname{mrad}\left(0.1^{\circ}\right)$.

The coordinates in the simulation are ruled by the tilt angle $\alpha_{\text {tilt }}$ and the deflection angle $\beta_{l}$ [Eq. (2)] and can be described by

$$
\begin{aligned}
\Delta x & =\frac{e \mu_{0} \lambda}{h} H_{z} \Delta y \equiv C_{1} H_{z} \Delta y, \\
\Delta z & =\Delta y \tan \left(\alpha_{\text {tilt }}\right)+\frac{e \mu_{0} \lambda}{h} H_{x} \Delta y \\
& \equiv \Delta y \tan \left(\alpha_{\text {tilt }}\right)+C_{1} H_{x} \Delta y,
\end{aligned}
$$

where $C_{1}$ is a constant that depends on the acceleration voltage. With the result of Eq. (2) and an electron energy of $100 \mathrm{keV}, C_{1}$ is estimated to be on the order of $10^{-13}$ $\mathrm{m} / \mathrm{kA}$. With these two dependencies (i.e., tilt and deflection) incorporated into the simulation program, the simulated equivalents of Figs. 10 and 11 resemble one another more closely. In Figs. 15 and 16 these equivalents are shown. The stray field values in Fig. 15 are in averaged values because they represent the average field that an electron experiences on its path.

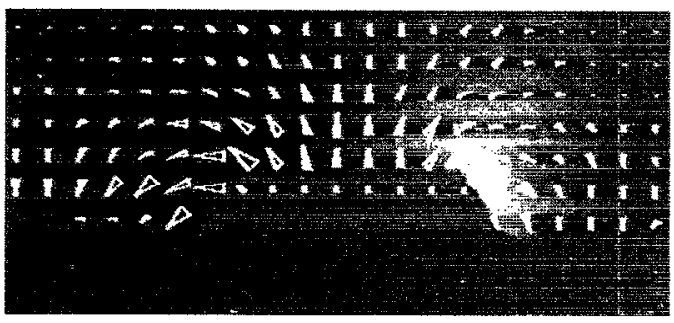

FIG. 11. Magnetic induction map of the sample No. 1. 


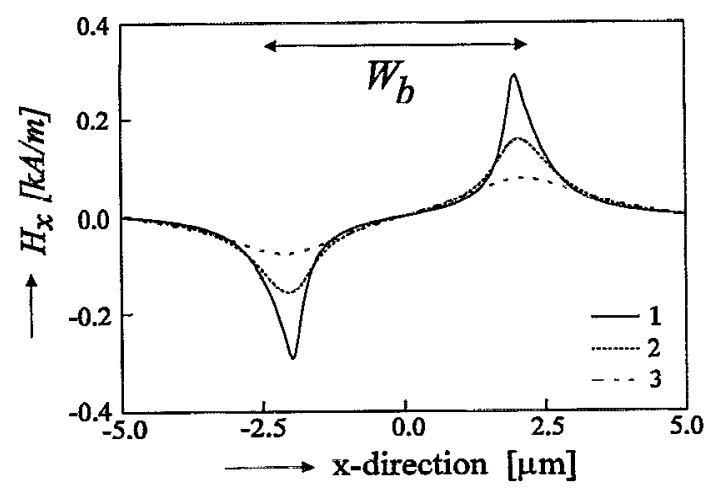

A

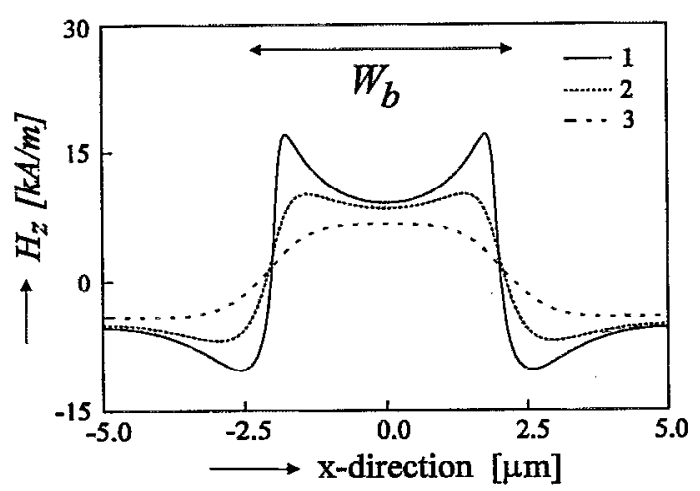

B

FIG. 12. Simulation of the mcasurcd line scans in Figs. 8 and 9 with a distance to the strip of $1=0.1,2=0.4,3=1 \mu \mathrm{m}$. (A) shows the $x$ component and (B) the $z$ component of the stray field. Parameters: $M r=91$ $\mathrm{kA} / \mathrm{m}$, width $=4.2 \mu \mathrm{m}$, period $10 \mu \mathrm{m}, \alpha_{1}=\alpha_{r}=30^{\circ}$.

It should be noted that both tilt and deflection constants have been obtained by trial and error. However, the chosen values result in deflection angles $\beta_{l}$ that are of the same order as those derived from the approximation in Eq. (2).

A tilt in the $z$ direction smoothes the stray field intensity, resulting in less sharp spikes in the stray field. A tilt in the $x$ direction and/or $C_{1}\langle\rangle 0$ results in sharper spikes but with an asymmetry with respect to the $H_{x}=0$ plane. The introduction of tilt and deflection in the simulation pro-

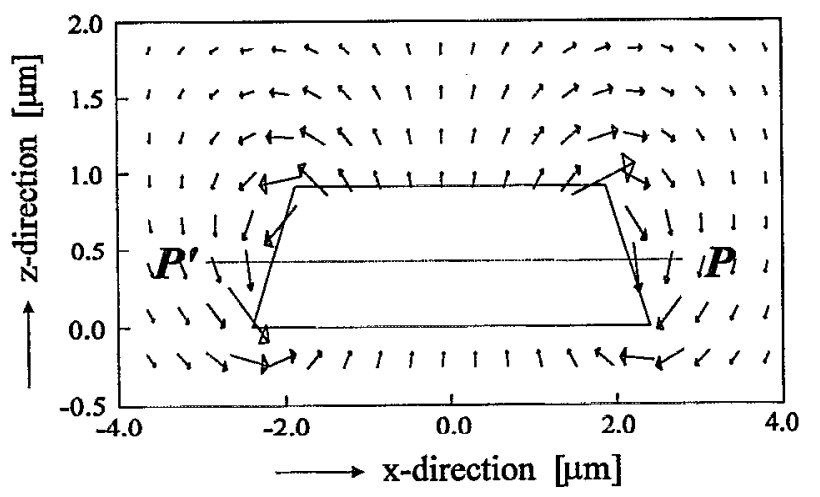

FIG. 13. Simulation of the measured induction map in Fig. 11. Paramcters: $M r=109 \mathrm{kA} / \mathrm{m}$, width $=4.8 \mu \mathrm{m}$, period $10 \mu \mathrm{m}, \alpha_{1}=\alpha_{r}=30^{\circ}$.

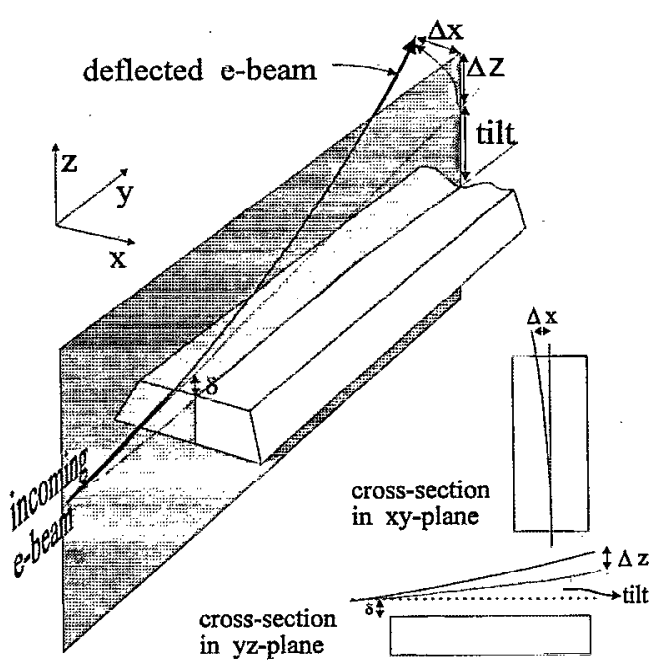

FIG. 14. Description of the tilt used in the simulation [see Eqs. (3) and (4)].

gram therefore offers better agreement between experiment and simulation. It also seems like a legitimate assumption and could possibly enhance the agreement between experiment and simulation in other work ${ }^{13,14}$ even further.
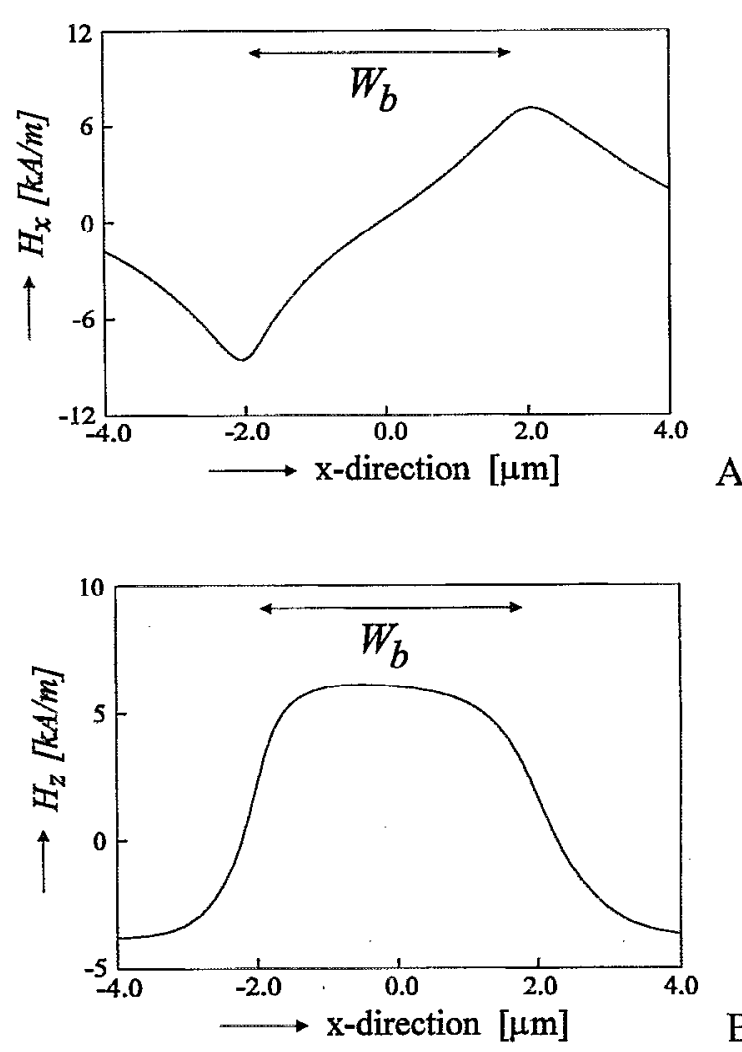

B

FIG. 15. Simulation of the measured line scans in Fig. 10, including tilt and deflection. Distance to strip: $0.2 \mu \mathrm{m}$. Parameters: tilt angle $=0.5^{\circ}$, deflection constant $C_{1}=5 e-14 \mathrm{~m} / \mathrm{kA}$. (A) shows the $x$ component and (B) the $z$ component of the stray field. 


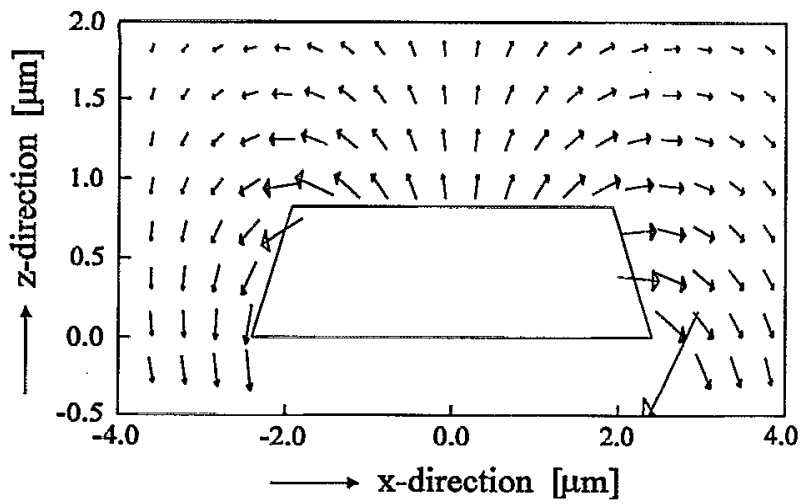

FIG. 16. Simulation of the measured stray field map in Fig. 11, including tilt and deflection. Same parameters as in Fig. 15.

\section{CONCLUSIONS}

We investigated the magnetic stray field of $\mathrm{Co}-\mathrm{Cr}$ microstrips with Foucault and DPC Lorentz electron microscopy. The Foucault mode operates simply and has high contrast. The DPC mode is more complicated but yields quantitative information. Both modes are, therefore, useful techniques to investigate magnetic stray fields.

The results obtained with the data processing are related to theory. Good agreement is obtained, which indicates that in a first approximation the assumed model gives a good representation of the stray fields coming from mag- netic strips. The assumed shape of the stray fields for bit stabilization by hard magnetic strips [Eq. (1)] has been verified for a distance $>1 \mu \mathrm{m}$.

\section{ACKNOWLEDGMENTS}

The authors would like to thank D. M. Donnet for assisting in the DPC measurements, and A. M. Otter for the SEM observations. This research has been financially supported by CAMST, a program of the European Community.

${ }^{1}$ S. Konishi, IEEE Trans. Mag. 19, 1838 (1983).

${ }^{2}$ P. Pougnet, L. Arnaud, M. Poirier, L. Zimmermann, M. Vaudaine, and F. Boileau, IEEE Trans. Magn. 27, 5492 (1991).

${ }^{3}$ G. Ronan, W. Glegg, and S. Konishi, J. de Phys. 46, C6-127 (1985).

${ }^{4}$ T. Suzuki, H. Asada, K. Matsuyama, E. Fujita, Y. Saegusa, K. Morikawa, K. Fujimoto, M. Shigenobu, K. Nakashi, H. Takamatsu, Y. Hidaka, and S. Konishi, IEEE Trans. Magn. 22, 784 (1986).

${ }^{5}$ Y. Maruyama, T. Ikeda, K. Fujimoto, and R. Suzuki, IEEE Trans. Magn. 24, 3027 (1988).

${ }^{6}$ J. G. Th. te Lintelo, J. C. Lodder, Th. J. A. Popma and J. Engemann, J. Magn. Magn. Mater. 115, 333 (1992).

${ }^{7} \mathrm{~J}$. C. Lodder and T. Wielinga, IEEE Trans. Magn. Magn. 20, 57 (1984).

${ }^{8}$ J. N. Chapman, J. Phys. D 17, 623 (1984).

${ }^{9} J$. N. Chapman, S. McVitie, and S. J. Hefferman, J. Appl. Phys. 69, 6078 (1991)

${ }^{10}$ J. Zweck, J. N Chapman, S. McVitie, and H. Hofmann, J. Magn. Magn. Mater. 104-107, 315 (1992).

${ }^{11}$ J. Simsova, R. Gemperle, and J. C. Lodder, J. Magn. Magn. Mater. 95, 85 (1991).

${ }^{12}$ J. Heidmann, R. Dahlbeck, H. Krause, and J. Engemann, IEEE Trans. Magn. Magn. 24, 3048 (1986).

${ }^{13} \mathrm{~J}$. Matsuda, K. Aoyagi, Y. Kondoh, M. Iizuka, and K. Mukasa, IEEE Trans. Magn. Magn. 26, 2061 (1990).

${ }^{14} \mathrm{M}$. Steck, H. Schewe, and E. Kubalek, IEEE Trans. Magn. Magn. 26, 1343 (1990). 